\title{
LA VOCATION AU CROISEMENT DES ESPACES DE SOCIALISATION
} Étude sociologique de la formation des footballeurs professionnels Julien Bertrand

\section{Presses de Sciences Po | Sociétés contemporaines}

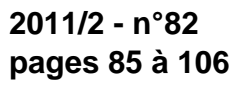

ISSN 1150-1944

Article disponible en ligne à l'adresse:

http://www.cairn.info/revue-societes-contemporaines-2011-2-page-85.htm

Pour citer cet article :

Bertrand Julien , « La vocation au croisement des espaces de socialisation » Étude sociologique de la formation des footballeurs professionnels,

Sociétés contemporaines, 2011/2 n82, p. 85-106. DOI : 10.3917/soco.082.0085

Distribution électronique Cairn.info pour Presses de Sciences Po.

(C) Presses de Sciences Po. Tous droits réservés pour tous pays.

La reproduction ou représentation de cet article, notamment par photocopie, n'est autorisée que dans les limites des conditions générales d'utilisation du site ou, le cas échéant, des conditions générales de la licence souscrite par votre établissement. Toute autre reproduction ou représentation, en tout ou partie, sous quelque forme et de quelque manière que ce soit, est interdite sauf accord préalable et écrit de l'éditeur, en dehors des cas prévus par la législation en vigueur en France. II est précisé que son stockage dans une base de données est également interdit. 


\section{Julien BERTRAND}

\section{La vocation au croisement des espaces de socialisation \\ Étude sociologique de la formation des footballeurs professionnels}

Le texte présente les résultats issus d'une enquête socio-ethnographique lentretiens, observations) à l'intérieur d'une structure qui prépare au métier de footballeur professionnel. Cet apprentissage exige un engagement intensif et la production d'une vocation à l'intérieur d'une organisation particulièrement enveloppante, le club professionnel. Cependant, cette conversion au métier sportif n'est pas indépendante des relations sociales nouées à l'extérieur du monde sportif, notamment à l'école, dans la famille et dans les réseaux de sociabilité. Ces relations, transformées par l'ascension sportive, apportent leur contribution à ce processus d'engagement.

a pratique du métier de footballeur est subordonnée, comme pour tous sportifs de haut niveau (Fleuriel, 2004) ou pour certaines pratiques artistiques d'excellence (comme les danseurs (Faure 2000)), à la réalisation d'un apprentissage intensif de longue haleine, qui seul peut donner les armes nécessaires à l'accès à un marché très concurrentiel. La vive mobilisation des jeunes aspirants se réalise à l'intérieur de structures, comme les centres de formation des clubs professionnels ${ }^{2}$, qui sont devenus depuis les années 1980 (Slimani, 2000) la voie privilégiée d'accès à la carrière. L'étude de l'un de ces centres (Bertrand, 2008) donne ainsi accès aux processus de socialisation à l'intérieur d'une organisation singulièrement enveloppante, et en particulier à la manière dont se construit progressivement un engagement intensif dans le football alors que les débouchées sont des plus incertains ${ }^{3}$. L'incertitude du devenir constitue, en effet, une dimension centrale de la condition d'apprenti footballeur étant donné le degré de sélectivité de ce marché du travail et la pression qu'entretient sur celui-ci l'abondance des aspirants. Cependant, il apparaît que la construction de cet engagement ne peut se comprendre réellement sans

1/ Je tiens à remercier les membres du comité du lecture qui, grâce à leur expertise, ont permis des améliorations sensibles de la version initiale du texte. Celui-ci a également bénéficié de la lecture avisée de Muriel Darmon, qu'elle en soit remerciée ici.

2/ On compte actuellement trente-deux centres de formation agréés. L'organisation distingue la « préformation » (12-14 ans) réalisée dans les centres fédéraux (douze pôles « espoirs » au total) ou parfois dans des clubs professionnels, et la «formation » proprement dite, qui est l'œuvre des centres de formation des clubs professionnels (CF).

3/ En 2005 par exemple, la Ligue du football professionnel comptabilisait 1960 joueurs sous contrat dont environ $47 \%$ étaient des jeunes sous contrat de formation. 
analyser en parallèle les autres « scènes sociales » (Weber, 1989) sur lesquelles circulent les apprentis footballeurs, dont en particulier les scènes familiales, scolaires et de sociabilité. Tout d'abord, c'est aussi dans ces différents espaces de relations que prend sens leur ascension sportive, d'où l'intérêt de les rapporter " aux univers socio-symboliques pertinents, c'est-à-dire à ceux dans lesquels ils sont effectivement vécus », (Schwartz, 1995: 296-297). Surtout, si l'on sait l'intérêt de l'étude de la pluralité des cadres de socialisation pour comprendre les appropriations des injonctions institutionnelles (comme le montre, par exemple, la «triple contrainte » (scolaire, familiale, amicale) qui pèse sur les adolescents « ordinaires » (Lahire, 2004), il prend une force singulière ici avec le degré d'exigence et de précocité de la formation sportive ${ }^{4}$. On peut, dès lors, faire l'hypothèse que l'entreprise sportive a d'autant plus de chance de succès qu'elle parvient à limiter les aspirations et investissements contradictoires des jeunes membres du club ou, autrement dit, qu'elle parvient à assurer une convergence suffisante avec les autres espaces d'appartenances des jeunes concernés. L'analyse des conditions de possibilité et des formes de cette convergence est ainsi rendu possible par l'ouverture de la focale d'observation. En d'autres termes, celle-ci permet de se demander dans quelle mesure la formation sportive prend, pour les enquêtés, la place de «plaque tournante » de la socialisation que l'école tend à assumer pour les adolescents ordinaires, c'est-à-dire à être « à la fois institution de socialisation spécifique et espace de mise en contact et d'articulation des autres formes de socialisation, voire institution évaluatrice des produits des autres instances de socialisation » (Darmon, 2006, 62). Après avoir décrit la force d'emprise de la socialisation interne au club, le texte s'attache ainsi à ainsi à préciser la manière dont s'articule ce parcours sportif et les autres investissements et relations sociales des jeunes enquêtés (école, famille, groupes de sociabilité).

\section{TERRAIN ET ENQUÊTE}

L'investigation qui fonde les analyses repose sur l'exploration intensive d'un centre de formation de l'un des principaux clubs français. L'enquête ethnographique, méthode peu employée sur ce terrain (Roderick, 2006), a été menée entre 2001 et 2004 à l'intérieur d'un club qui sélectionne et forme environ cent trente jeunes footballeurs âgés de 12 à 19 ans. "Amateurs » lors des premières années,

4/ Bien que l'apprentissage du football reste nettement moins chronophage et précoce que d'autres pratiques sportives (telles la gymnastique (Papin, 2007)) ou artistiques (Wagner, 2004). 
la plupart d'entre eux signe un contrat de formation. Dès l'âge de 17 et 18 ans ce sont environ huit apprentis sur dix qui sont liés par un contrat avec le club /d'une durée d'un à trois ans) selon les règles édifiées dans la Charte du football professionnel.

Trois principaux matériaux sont à l'origine des analyses. 58 entretiens ont tout d'abord été réalisés. 33 ont été faits avec des aspirants professionnels âgés de 16 à 19 ans et ont été complétés par l'interview de parents (4) et d'anciens pensionnaires du Centre (2). De plus, la quasi-totalité des intervenants sportifs, médicaux, scolaires, de la structure ont été rencontrés $(n=16)$. Une observation prolongée à l'intérieur du club a constitué le deuxième volet de l'enquête. Environ 200 séances d'observation ont été menées à l'intérieur du club (soit entre 500 et 600 heures de présence dans la structure) auprès d'équipes situées en début et en fin de cursus. Si les terrains de jeux sont restés les lieux les plus accessibles (entraînements et matchs), d'autres espaces sportifs ont pu être progressivement et partiellement investis (vestiaires, salle de soin), tout comme un de leur lycée d'accueil (en tant que surveillant durant une année). Enfin, l'analyse de 36 dossiers scolaires a permis de renforcer le degré d'objectivation des parcours des joueurs. Leur reconstruction s'est appuyée sur le recueil des données disponibles dans les bulletins : l'itinéraire scolaire (orientation, redoublements, diplômes), les notes moyennes annuelles (moyenne générale et d'EPS) et leur écart à la moyenne de la classe, les sanctions positives et négatives décernées par les conseils de classe ("Félicitations 》, "Avertissements 》, etc.), les « observations » récurrentes des enseignants et les absences.

\section{ENTRÉE DANS LE CLUB ET PRODUCTION D'UNENGAGEMENT INTENSIF}

\section{- La formation : quand le football devient une préoccupation centrale}

En entrant dans la structure de formation, soit en moyenne entre 13 et 14 ans, notre population d'apprentis footballeurs est progressivement immergée dans un espace orienté vers la performance sportive. Ils pénètrent dans une organisation enveloppante qui inscrit les apprentis dans un «contre-espace » et un «contre-temps» (Faure \& Suaud, 1999: 199) qui tend à devenir de plus en plus « leur» monde. Les jeunes apprentis intègrent comme lieu de vie (plus de la moitié sont internes), ou comme lieu de pratique, un espace construit autour de sa fonction sportive et dont la centralité s'accroît à mesure que la formation entraîne l'inflation du temps d'entraînement (on passe de 4 à 7 ou 8 séances hebdomadaires auxquelles s'ajoute le temps de compétition). De plus, réunis avec des pairs aux aspirations semblables, ils connaissent une intensification de la pratique que permettent des aménagements de scolarité (les horaires sont adaptés au collège et au sein du lycée professionnel, ceux qui s'orientent vers le baccalauréat le préparent en quatre années). Leur concentration 
autour des enjeux sportifs est, enfin, renforcée par l'organisation sélective et hiérarchisée de la formation. Écrémage progressif de la population (lors des premières années de préformation par exemple, un quart à un tiers des équipes est renouvelé en fin de saison) et rareté des places disponibles au sein des équipes participant aux compétitions font de la sélection et de la concurrence interne une constante de l'expérience d'apprenti (Bertrand, 2009). La structure a ainsi toutes les chances de produire des êtres tendus vers la performance sportive, toujours pris dans la préparation de la prochaine échéance footballistique, appelés à faire un usage intensif de leur temps et à intérioriser un rapport ascétique à l'effort. Cette insertion transforme la vie des apprentis et tend à les faire adhérer sur le modèle de la "vocation », mode d'engagement intensif légitimé et produit par cet espace social. Toute l'organisation sportive fait, en effet, de la «passion » le mode légitime d'engagement sur cette voie. Comme dans nombre de métiers dits de vocation (en particulier artistique ou religieuse (Suaud, 1978)), le rapport des enquêtés à la formation tend à cumuler trois propriétés caractéristiques : un attachement personnel intense qui se manifeste par le sentiment d'être « fait pour ça », généralement vécu par l'intermédiaire de la catégorie du «don» ou du «talent» (à forte valeur personnelle) et qui tend à dénier la genèse sociale de ce type d'engagement; la conviction de s'engager dans une activité qui n'est pas ordinaire et qui ne peut être totalement assimilée à un «travail » (à forte valeur symbolique) et que les apprentis opposent volontiers à l'école et à ses contraintes ; et enfin un investissement intense, que vient légitimer le langage de la passion (à fort investissement), et qui rend poreuse la frontière entre activité professionnelle et sphère privée. Or, les jeunes footballeurs sont d'autant plus portés à vivre de la sorte leur engagement que leur entrée dans le club constitue l'aboutissement d'une pratique amateur longue et sélective. Familiarisés précocement au jeu (plus de la moitié des joueurs enquêtés sont entrés dans un club à quatre ou cinq ans), ils ont connu une ascension sportive par laquelle, à force d'élections et de gratifications, ils ont acquis le sentiment d'être "doués». Ainsi, dès l'âge de onze ans les deux tiers d'entre eux s'entraînent déjà dans des clubs du haut de la hiérarchie sportive. Cette intégration des jeunes apprentis dans l'univers footballistique a donc d'autant plus de chances de succès qu'elle s'adresse à une population déjà fortement sélectionnée.

\section{Le football à corps perdu}

La structure organise donc le centrage progressif des apprentis vers les performances sportives au point que cet investissement constitue 
pour eux une véritable « affaire personnelle » et une partie du sens de leur existence. Cet engagement se concrétise de manière particulièrement nette dans les usages des corps auxquels sont familiarisés les jeunes enquêtés. Ils illustrent cette appropriation du métier et de ses contraintes, de l'exigence de mettre son corps au service des performances sportives. En effet, en avançant dans le cursus, les apprentis intériorisent un rapport au corps fait tout à la fois d'une exigence à son égard, fondée sur l'injonction à « dépasser » les douleurs, et d'une attention fébrile à ce capital. L'apprentissage d'un rapport instrumental au corps est ainsi marqué par cette double orientation. D’une part, les apprentis apprennent à faire une utilisation maximale des ressources physiques pour faire face à l'urgence sportive, usage qui implique de faire preuve d'un corps résistant et sans cesse disponible pour l'exercice sportif. La tendance des joueurs à dissimuler certaines douleurs au corps médical du club pour faire prévaloir le maintien dans l'activité est une manifestation récurrente de lintériorisation de linjonction répétée par les entraîneurs à «ne pas trop s'écouter ». Aussi face à de nombreuses sensations douloureuses, le premier réflexe des apprentis est bien souvent d'attendre, de taire la douleur en espérant qu'elle « passe ». Et cela d'autant plus que pour eux la déclaration de leur douleur auprès des soignants du club enferme le risque d'une dévalorisation, d'être « mal vu», de « passer pour une chochotte».

D'autre part, et de manière complémentaire, les jeunes joueurs développent une conscience aiguë de la fragilité de leur capital corporel. Le kinésithérapeute du club est le premier témoin de cette inquiétude spécifique: "Dès qu'ils sont blessés, ils aimeraient savoir deux heures après dans combien de jours et dans combien de minutes ils vont reprendre. C'est ça le plus difficile, c'est de donner la date de reprise et de mettre en confiance le joueur parce que le sportif, c'est un mec dès qu'il est blessé c'est la catastrophe. Donc il faut vraiment le rassurer. (...). La grande différence (avec ses patients ordinaires), c'est l'exigence des résultats. Au cabinet, les patients sont moins inquiets. Beaucoup moins inquiets ». Ils acquièrent ainsi un souci de la préservation de leurs ressources physiques. Ce souci, que véhicule le personnel médical et la structure même de la pratique - chaque séance d'entraînement s'organisant systématiquement autour de la même succession d'exercices et de soins du corps (échauffement/reposhydratation/étirement) -, se traduit dans la constitution d'un véritable « sens de l'épargne corporelle (Wacquant, 1989). Celui-ci se concrétise dans une série de pratiques d'entretien et d'économie du corps qui deviennent constitutives de leur mode de vie. En effet, cet apprentissage de la préservation des forces s'actualise au sein même de la pratique (par le souci de la gestion des efforts) mais également en périphérie du jeu par des renonciations (attention alimentaire, 
abstention de sports "à risques ») ou des pratiques de soins et de récupération qui se généralisent (sieste, prises fréquentes d'adjuvants comme des vitamines - environ deux tiers des enquêtés déclarent en consommer parfois pour faire face à un «coup de fatigue » ou pour se donner un « coup de fouet »).

Ces usages

du corps,

qui articulent

dépassement

et écoute

instrumentale

du corps,

témoignent de

I'intériorisation

des exigences

d'une adhésion

vocationnelle au projet sportif.

Ces usages du corps, qui articulent dépassement et écoute instrumentale du corps, témoignent de l'intériorisation des exigences d'une adhésion vocationnelle au projet sportif. Mais ce sont peut-être les mots de cet ancien pensionnaire du centre, membre du club durant six ans et reconverti depuis plusieurs années dans un autre domaine professionnel, qui illustrent le mieux ce processus d'absorption par des enjeux spécifiques. Il décrit son expérience à partir d'une opposition très significative entre la vie « réelle», «normale », le «vrai » monde, et l'existence «irréelle», «déconnectée» de l'apprenti footballeur. La vie d'aspirant se distingue selon lui par son caractère exceptionnel et par l'emprise de cet espace et de ses enjeux, lui faisant dire qu'il vivait dans une «bulle», dans un «microcosme»: «le football, c'est ce qui te fait aller, c'est ce qui te fait vivre, ce qui te fait être bien, pas bien. (...). On est hors du temps quoi ». Sa sortie de l'univers footballistique lui permet une distance face à cet engagement (« on se rend pas compte », dit-il souvent pour évoquer cette époque) et le porte à considérer cette vie comme presque étrangère à lui-même. Cette impression de mener une vie à part, à l'intérieur d'un monde clos, souvent soulignée chez les sportifs de haut niveau, est révélatrice du degré d'emprise de cette «bulle » qu'entraîne la socialisation footballistique.

L'apprentissage footballistique se révèle donc être une socialisation puissante, et cela d'autant plus qu'elle peut s'appuyer sur une familiarisation précoce au football. La construction de cet engagement ne peut, cependant, être dissociée de la place que ces jeunes viennent à occuper sur une autre scène centrale : l'école.

\section{L'ÉCOLE ET LES CONTRAINTES D'UNE DOUBLE FORMATION}

\section{Une concurrence croissante}

Du fait de sa précocité, la formation footballistique s'articule nécessairement avec l'apprentissage scolaire. Or, même si le club investit dans l'organisation scolaire (par l'emploi d'un responsable 
pédagogique par exemple $)^{5}$, l'analyse des parcours scolaires montre l'établissement d'une concurrence croissante entre les deux cursus.

Du côté des formateurs sportifs tout d'abord, le souci scolaire décroît à mesure que les jeunes concernés avancent dans le cursus. La surveillance très active auprès des jeunes de 12 ans recule très nettement en fin de cursus, au grand regret du responsable pédagogique, notamment lorsqu'elle est prise en charge par d'anciens joueurs professionnels. D'autre part, les données produites permettent également de souligner les effets de cette entrée dans une vie « à part » sur l'investissement scolaire des appentis. Cette population ne se caractérise pas par des difficultés scolaires particulières au moment de l'entrée en formation. Notons, par exemple, qu'en fin de cycle primaire, seulement trois d'entre eux (sur 43 cas) accusaient un retard (d'une année). Il faut dire que si la formation continue d'attirer davantage d'enfants issus des milieux populaires $(57,5 \%$ de pères ouvriers ou employés), elle est loin d'exclure les catégories sociales moyennes et supérieures (près d'un sur cinq ont un père appartenant à la catégorie des cadres et professions intellectuelles supérieures) ${ }^{6}$, notamment $\mathrm{du}$ fait d'une organisation qui rend compatible et crédible la double formation sportive et scolaire ${ }^{7}$. Cependant, l'étude de l'implication et résultats des enquêtés témoigne d'une tendance à la baisse de leur investissement, principalement à partir de l'âge de 15 ans. Si l'intensité et les effets de celle-ci dépendent de dispositions scolaires préalables et socialement situées, son effet est relativement généralisé. Les orientations ou réorientations vers des filières jugées plus faciles (le baccalauréat technologique), la baisse objective des résultats en témoignent. Au lycée général et technologique, un seul enquêté voit ses notes progresser entre le début et la fin de ce cycle et la diminution des moyennes générales affecte la majorité des enquêtés. Cette baisse est encore légère lors des trois premières années mais les sanctions des conseils de classe sont elles beaucoup plus nombreuses (de 3 à 16 « avertissements »). La chute s'accélère sensiblement lors de la classe de terminale (avec une moyenne en baisse de 2,6 points par rapport à la classe de seconde) alors que la proportion de sanctions négatives par

5/ Cet investissement, qui est central dans la légitimation sociale de la formation, s'explique également par la concurrence que se livrent les clubs pour attirer les meilleurs éléments.

6/ Ce recrutement reste plus populaire que celui des sportifs de haut niveau (Fleuriel, 2004, 76). Notre population possède des propriétés sociales très proches de celle relevée par les travaux statistiques réalisés au milieu des années 1990 sur les apprentis et footballeurs professionnels (Slimani, 2000).

7/ On peut faire l'hypothèse que plus la formation sportive est exclusive plus elle n'atteint que les publics populaires. C'est le même constat qui a pu être fait dans le cas de la formation au cyclisme (Lefèvre, 2007, 290). On peut ainsi faire le lien entre la généralisation des centres de formation et l'ouverture du recrutement aux classes supérieures : en 1996, les enfants de cadres et professions intellectuelles supérieures représentaient environ 13,3\% des footballeurs professionnels contre moins de 4 \% en 1983 (Faure \& Suaud, 1999 , 219-220). 
élève reste tout aussi élevée. Au final, environ $56 \%$ des apprentis ont obtenu le BEP ou un baccalauréat (environ un tiers) au terme de leur cursus (39 cas). Dans un contexte d'intensification des contraintes sportives, l'école tend à devenir une contrainte concurrente. Ce mouvement s'amplifie à la fin du cursus de formation et l'arrêt de la formation scolaire après la classe de terminale, c'està-dire pour la dernière année d'apprentissage au Centre, devient une norme largement répandue parmi les joueurs du club. L'expression, récurrente en fin de formation, d'une lassitude à l'égard de l'école ( «jai de moins en moins envie » disent-ils souvent), le sentiment d'avoir de moins en moins «la tête à ça », le souhait de ne plus «se prendre la tête avec ça» sont les témoins de leur absorption progressive par les enjeux sportifs et d'une baisse des attentes scolaires d'autant plus marquée que la majorité des aspirants sont issus de familles populaires aux capitaux scolaires modestes ${ }^{8}$. Enfin, la difficulté pour la grande majorité d'entre eux à se projeter dans un univers professionnel non-sportif (c'est-à-dire autre que joueur, entraîneur, professeur d'EPS, kinésithérapeute, etc.) témoigne de la focalisation de leurs aspirations et alimente, de façon circulaire, la tendance au retrait progressif d'un avenir scolairement pensé ${ }^{9}$.

Si les performances scolaires sont fortement contrastées à l'intérieur du Centre, ce que l'on peut relier à la relative diversité sociale $\mathrm{du}$ recrutement, l'analyse des parcours montre une évolution commune et sensible de leur rapport à l'école. Ayant des passés et des dispositions scolaires inégales, cette tendance n'a pas les mêmes conséquences pour tous: alors que les élèves les plus en difficulté abandonnent parfois la formation scolaire, ceux qui disposaient d'acquis importants poursuivent leur cursus tout en voyant leurs résultats diminuer. On peut noter, par exemple, que dans le groupe d'enquêtés qui ont opté pour un baccalauréat général, les enfants d'ouvriers sont nettement sous-représentés (environ 1 sur 5). Cependant, produite par la concurrence entre les deux formations, la modification de la forme de leur appartenance à l'univers scolaire se lit au-delà de leurs résultats scolaires. C'est également la place spécifique et les relations nouées dans cet espace qui participent à la construction de ces itinéraires.

8/ Même si les enquêtés connaissent mal les diplômes parentaux (ce qui, on peut le supposer, est déjà un indicateur de leur faiblesse), les entretiens révèlent que les trois quart des parents n'ont pas atteint le niveau du baccalauréat.

9/ D'autres travaux ont pu montrer de quelle manière l'engagement vocationnel dans une activité peut rendre difficile la projection dans un autre univers professionnel (Sorignet, 2004). 


\section{Une place à part dans l'espace scolaire : la force du « marquage » symbolique}

La relation entre les deux formations, sportives et scolaires, se traduit également dans la manière dont les apprentis footballeurs s'insèrent dans l'espace des relations scolaires. Quoi qu'en disent les responsables du club ou des établissements, lorsqu'ils affirment ne pas vouloir créer un " ghetto " (responsable pédagogique du centre de formation) et les traiter comme des élèves « comme les autres », plusieurs éléments différencient les joueurs dans les établissements. Ils tendent à être placés dans une position spécifique qui a toutes les chances de les amener à s'approprier l'espace scolaire en tant que footballeurs. Tout d'abord, en entrant dans les établissements scolaires, les apprentis footballeurs y importent leurs capitaux propres. Appartenant au plus grand club de football de la région et par là-même à l'élite du sport le plus pratiqué par cette classe d'âge, ils sont porteurs d'un capital symbolique hautement distinctif. Certains articles de presse narrant leur parcours sont d'ailleurs parfois exposés dans les établissements, comme dans le lycée général où ils sont scolarisés. De plus, leurs revenus leur permettent des dépenses fortement significatives dans cet espace (voitures, téléphones portables dernier cri, lecteurs MP3, vêtements de marque, etc.). Ces attributs viennent redoubler une spécificité qui est, en réalité, déjà produite par les aménagements scolaires dont ils jouissent. Ils bénéficient d'un traitement différencié (aménagement du temps scolaire et classes spécifiques) qu'ils partagent la plupart du temps avec d'autres « sport-étude » intégrés dans ces établissements, et qui traduit en acte la reconnaissance de leur particularité par l'institution scolaire. À cette position inscrite dans les règlements, s'ajoute souvent l'effet de leur statut sur le personnel des établissements. Les enquêtés relatent souvent, en effet, le sentiment de bénéficier, dans un sens positif ou négatif, d'un traitement relativement différencié dans leur lycée. Selon leurs dires, leur parcours suscite auprès des agents scolaires curiosité, attraction ou, au contraire, méfiance. Certains estiment que les «profs sont plus indulgents », qu'ils sont eux «plus privilégiés» ou regrettent d'avoir été «catalogué ». Quelles que soient les réactions, ils trouvent dans leurs interactions avec le personnel des établissements la confirmation qu'ils ne sont pas des élèves ordinaires et que leur engagement, jugé légitime ou non, les distingue. Affectés dans ces établissements en raison de leur engagement sportif, bénéficiant d'un statut particulier en leur sein, ils occupent à l'école une place dépendante de leur appartenance au club dont les effets sont sensibles sur leur sociabilité scolaire. 


\section{La « jalousie » dans le lycée : les effets du capital symbolique}

Il est notable que la visibilité de leur statut au sein du lycée est très fréquemment associée à la crainte d'y être mal-aimé et d'être pris pour cible de railleries ou provocations. La renommée qu'ils importent dans l'espace scolaire produit des effets ambivalents et le sentiment de devoir parfois faire face à des réactions hostiles est très répandu chez les enquêtés. Il n'est pas rare que les joueurs s'y sentent peu à l'aise. Les observations confirment d'ailleurs l'existence de bravades, généralement initiées par les lycéens les plus déclassés dans l'espace scolaire (ceux appartenant aux sections dévalorisées des filières professionnelles), comme ce groupe de jeunes criant, au passage de joueurs, le nom du club professionnel voisin et rival historique de celui des enquêtés. Si la perception et la reconnaissance de ces capitaux par les élèves non-sportifs paraît hétérogène, la vision qu'en ont les joueurs est, elle, unanime : ils considèrent systématiquement que les lycéens « ordinaires» ne les «aiment pas». Ils partagent ce qu'ils ressentent comme un discrédit injuste, qui prend le plus souvent la forme de remarques accusatrices ( « avoir la grosse tête », "faire les malins ») ou d'insultes, et qui dépassent rarement la provocation verbale. Pour les enquêtés, l'hostilité à leur égard se résume en grande partie à des accusations de fierté qu'ils interprètent comme le signe d'une « jalousie » qui se cristallisent sur les questions des aménagements scolaires dont ils sont les bénéficiaires et leurs relations avec les lycéennes. Cette perception est d'autant plus commune qu'elle les conforte dans leur conviction, constitutive de la vocation, que résume l'un d'entre eux: «Y en a qui sont jaloux parce qu'ils auraient bien aimé faire comme nous ». Grâce au pouvoir de leurs capitaux sportifs, y compris au sein de l'espace scolaire, ils ne peuvent percevoir cette conflictualité que comme une reconnaissance, qui se nie elle-même, de leur valeur et de leur « chance » d'être où ils sont et qui ils sont. Cette impression participe à la tendance de leur sociabilité lycéenne à se limiter à un entre-soi sportif qui fait alors office de refuge (Bertrand, 2009b). Cette propension est suffisamment forte pour qu'elle se concrétise par une appropriation spécifique de l'espace, les sportifs ayant l'habitude de se retrouver dans les mêmes lieux au sein du lycée, dans ce qu'ils nomment le « coin des sportifs ${ }^{10}$.

Ainsi, leurs relations scolaires, parce qu'elles s'inscrivent prioritairement dans le prolongement de leur affinités sportives, favorisent

10/ On peut noter que si cette sociabilité élective est portée par une tendance à l'« homophilie » (c'està-dire « comme la tendance, pour les amitiés, à se former entre individus qui se ressemblent sur un aspect précis » (Bidart, 1997, 42), elle est également défensive parce qu'elle est une façon pour eux d'éviter les atteintes dont sont potentiellement porteurs une partie de leurs camarades. 
une appropriation collective et spécifique de l'espace scolaire. C'est de cette manière que leur scolarisation, pourtant réalisée au sein d'établissements extérieurs, apporte sa contribution propre à l'engagement sportif. Les effets de la formation sportive sur les parcours scolaires sont donc doubles: elle affecte l'investissement dans les études et modifie leur place à l'intérieur des relations scolaires.

\section{$L^{\prime}$ «ÉLU » SPORTIF PARMI LES SIENS : LAMOBILISATION FAMILIALE}

L'intervention du club dans les parcours scolaires témoigne de la prise en charge élargie qu'il opère et de son action dans des domaines qui relèvent d'ordinaire de l'intervention parentale. Mais si, pour une part, la formation opère une mise à distance de la famille (séparation familiale avec l'internat, prise en charge élargie), la réalisation de la formation s'appuie, le plus souvent, sur un engagement familial qui, de diverses manières, contribue à la focalisation autour des enjeux sportifs.

\section{L'appropriation familiale du projet sportif}

Les parents jouent, le plus souvent, un rôle notoire dans la prime inculcation de la «passion » sportive. Les membres du Centre ont fréquemment été initiés à la pratique par un père lui-même pratiquant: deux tiers des pères ont joué en club et un tiers dans des championnats nationaux ${ }^{11}$. L'imprégnation par une culture footballistique au sein de familles investies dans cette pratique (notons, par exemple, que neuf frères sur dix des enquêtés ont eux-même pratiqué ce jeu) constitue un déterminant important de ces parcours. Et cette imprégnation a d'autant plus de chances d'être forte que l'on connaît la place que tient le football dans les styles de vie masculins dans la partie inférieure de l'espace social.

Avec l'entrée en formation, l'engagement parental s'accroît progressivement même si cette mobilisation a pu être, dans un premier temps, freinée pour des raisons scolaires. En effet, les récits des joueurs et des parents montrent bien que la question scolaire est un motif fréquent d'hésitations parentales, et notamment maternelle, à l'entrée en formation. Du fait de la précocité du recrutement du club, la question de l'organisation de la scolarité s'est fortement posée dans

11/ C'est-à-dire entre les divisions professionnelles et le niveau de CFA2 (Championnat de France Amateur 2), dernière division nationale dans la hiérarchie (Ligue 1, Ligue 2, National, CFA, CFA 2). 
la grande majorité des familles et l'accord semble avoir souvent été déterminé par la croyance des parents dans la capacité du Centre à offrir des conditions favorables à la scolarité de leur enfant. Certains des parents, particulièrement les membres des classes supérieures ou moyennes, font d'ailleurs état de leurs primes réticences et de leurs tentatives pour imposer leurs conditions scolaires pour que le recrutement ait lieu. C'est le cas de ce père enseignant, par exemple, qui n'a accepté l'orientation vers le club qu'une fois rassuré par l'organisation mise en place: "En aucun cas il n'aurait été à X [le club] si ses études en avaient souffert d'une manière ou d'une autre. C'était la condition. C'était la condition donc le club a fait ce qu'il fallait puisque pour l'emmener au collège, (...) ils nous ont quand même affrété un taxi pendant deux ans tous les matins, (...) ils ont mis des moyens pour que Paul puisse être dans de bonnes conditions». Comme l'illustre ce cas, la question scolaire cristallise la plus grande distance de ces familles à la professionnalité sportive. Malgré ces réticences, le suivi familial des prestations sportives est souvent intense. Leur intérêt pour le parcours sportif se traduit d'abord par leur assiduité aux rencontres footballistiques, représentant parfois un investissement temporel et financier non-négligeable. L'agenda sportif est approprié par les familles et l'attention croissante à celui-ci se traduit par l'extension de l'intérêt pour les rencontres: les mères deviennent nettement plus assidues aux rencontres et celles-ci attirent parfois des membres de la famille élargie (grands-parents, oncles, cousins, etc.). De manière générale, les performances des enquêtés tendent à devenir une « affaire familiale » et le sujet récurrent de curiosité à l'intérieur de cette configuration de relations. Attention et mobilisation façonnent une place distinctive pour les jeunes apprentis qui contribue, parfois, à en faire des modèles d'identification à l'intérieur de la fratrie. Il faut noter, enfin, que si l'inflation de l'intérêt familial se traduit souvent par une mobilisation élargie, elle n'efface pas les différenciations sexuées et le père continue d'être, surtout lorsqu'il est un ancien pratiquant, au centre d'une relation privilégiée nouée autour des succès sportifs. Nombreux sont ainsi les apprentis qui entretiennent une relation très étroite avec leur père, dans laquelle les conversations footballistiques prennent une place centrale ${ }^{12}$. S'observe ici la permanence du rôle du père comme référent et la dimension affective de l'investissement footballistique des apprentis, investissement capable de combler les attentes de ces pères fortement mobilisés.

12/ Il existe souvent une distribution sexuée forte : le père, jugé plus compétent, est le partenaire privilégié des discussions footballistiques, alors que les mères sont plus souvent sollicitées pour l'extra-sportif (dont l'école) ou les dimensions moins spécifiques de l'activité. De manière générale, les réticences les plus marquées à l'égard de la formation semblent être le fait des mères appartenant aux classes moyennes et supérieures. 


\section{La famille : espace d'attentes et espace de repli}

La mobilisation fréquente des familles autour du projet sportif contribue à dessiner une place singulière et valorisée pour ces jeunes au sein de leur famille et renforce ainsi l'engagement dans cette voie des apprentis puisque c'est un des mécanismes les plus puissants de l'ascension sportive que d'agir sur le regard que portent les parents. Les élections sportives agissent, tels les rites d'institution, sur «la représentation que la personne se fait d'elle-même et les comportements qu'elle se croit tenue d'adopter pour se conformer à cette représentation », mais également sur « la perception que s'en font les autres agents et surtout peut-être les comportements qu'ils adoptent à son égard » (Bourdieu, 1982, 59). Elles facilitent ainsi la production de nouvelles attentes qui sont porteuses d'une injonction à «être à la hauteur » de ces espérances et de ces investissements. Ce mécanisme est particulièrement visible lorsque se cumulent déclin sportif et forte implication familiale. Par exemple, la crainte d'Éric de ne pas accéder à l'élite est intiment mêlée à celle de ne pas combler les espoirs parentaux : «Avant il fallait que moi je sois parfait quoi, que je loupe rien du tout. Mais moi j'ai horreur de... un peu qu'on soit déçu de moi. (...). Mais je veux pas décevoir c'est tout. Même au niveau des gens qui me soutiennent et tout, je sais que tant que c'est pas fini je lâcherai pas quoi. Il faut que je reparte du bon pied quoi, faut pas que je déçoive les gens qui ont cru en moi, moi c'est surtout ça moi. (...) Moi ce que je voudrais surtout pas, c'est décevoir les gens comme mes parents, tout ce qu'ils ont fait (...). Même si j'y arrive pas, y aura pas de problème, mais même je serai déçu quand même. Par rapport à eux de pas, de pas avoir fait le maximum, de pas y être arrivé. Déjà par rapport à moi et pour les gens qui ont cru en moi quoi. C'est tout. Même si je sais que c'est pas facile, je m'accroche » [17 ans, élève de première STT et fils d'un ouvrier, ancien pratiquant et entraîneur, et d'une employée]. De plus, la place des jeunes footballeurs dans la famille se singularise aussi par les revenus financiers qu'ils tirent de leur activité. Il est fréquent, environ la moitié des enquêtés déclarent le faire ou l'avoir fait, que les apprentis reversent une partie de leur salaire à leurs parents (essentiellement dans les familles populaires). Leur participation précoce à la santé économique de la famille accroît alors leur position spécifique à l'intérieur de celle-ci.

La mobilisation familiale constitue également une ressource nonnégligeable pour faire face aux aléas de la formation. Celle-ci les confronte, en effet, à un univers de forte concurrence et d'incertitudes. Sanctions des entraîneurs, contre-performances, blessures, sont autant d'événements qui peuvent menacer même les «meilleurs » d'entre eux. D'où cette tendance dans leurs récits à décrire le «chez soi » 
comme un lieu possible de repli, sécurisé, relativement à l'abri des atteintes symboliques que contiennent les aléas sportifs. Pour faire face aux doutes qui les assaillent après de mauvaises performances, la famille est souvent appelée à jouer un rôle central, celui d'un contreespace protecteur, un «cocon» dit l'un d'entre eux, où l'on peut « oublier» et se faire «réconforter». La famille proche fonctionne donc comme un lieu de refuge affectif sans lequel la poursuite de l'engagement serait plus délicat. Ainsi, qu'ils soient apprentis comme ici ou déjà professionnels (Faure \& Suaud, 1999 : 235), les footballeurs sont confrontés à un contexte marqué par l'incertitude, la concurrence et l'individualisation des carrières qui fait de la sphère privée le lieu de repli privilégié où l'on panse les plaies de la carrière sportive. De plus, cette place de la famille est renforcée par le fait qu'elle offre souvent l'occasion de suspensions provisoires des astreintes. Cette dimension est particulièrement visible chez les internes qui évoquent souvent les retours au domicile familial (weekends, vacances) comme un temps où ils peuvent davantage « en profiter », « se lâcher ». Les plaisirs de ces retrouvailles culinaires, le goût réactualisé de plats antithétiques avec les règles de la diététique sportive, constituent alors une licence provisoire, un renversement temporaire de l'ordre alimentaire sportif ordinaire. Cette rupture est vécue comme une compensation, par le plaisir du goût et de l'abondance, aux efforts consentis et peut prendre le sens d'une réaffirmation en acte de l'appartenance familiale commune ${ }^{13}$.

Les relations familiales sont donc souvent affectées par l'ascension sportive et dessinent ainsi une place spécifique à ces fils « doués ». On perçoit, de plus, que l'engagement a davantage de chances de se consolider à l'intérieur de familles mobilisée autour de la cause sportive, familles qui se mettent d'autant plus au service de la carrière sportive qu'elles sont proches de l'univers footballistique, qu'elles peuvent y trouver un complément financier (surtout dans les familles populaires) et que la formation ne rentre pas en forte contradiction avec leurs attentes scolaires.

\section{SOCIABILITÉ, GROUPE DE PAIRS ET RELATIONS D'INTERCONNAISSANCES LOCALES}

\section{«Passer à côté de sa jeunesse »}

Face à cette mobilisation familiale et soucieux de préserver l'autonomie de leurs jugements et leur pouvoir sur les carrières des

13/ Surtout lorsqu'on connaît le rôle toujours important des repas dans la vie familiale (Régnier et al., 2006, 39-40) 
apprentis, les formateurs sportifs témoignent souvent d'une certaine méfiance à l'égard des parents et leur réserve une place périphérique. Cependant, il existe un domaine qui concentre leurs craintes d'un détournement des apprentis de l'effort sportif : les relations amicales et certaines activités associées à leur classe d'âge. On comprend d'autant mieux cette crainte que l'on sait que, à l'âge où les membres du club perfectionnent leurs savoir-faire footballistiques, les groupes de pairs et de relations générationnelles donnent lieu à une sociabilité intense dont le poids se mesure, par exemple, dans l'évolution des pratiques culturelles ${ }^{14}$. Cependant, l'analyse montre que sur ce terrain aussi l'engagement sportif modifie l'espace de relations et que la formation sportive place les jeunes footballeurs dans une position singulière. Tout d'abord, à l'image de ce qu'ils connaissent dans leur famille, les relations de sociabilité constituent un espace de consécration. Parmi les relations extérieures au club, leur carrière est fréquemment l'objet d'une attention particulière et d'une curiosité soutenue. Elle constitue, de plus, une forme de capital attractif. Comme l'illustre le récit d'un des enquêtés, connaître un joueur du club professionnel peut constituer un motif de fierté : «Y avait les 40 ans du club de chez moi là, cet été-là. Donc il fallait qu'on fasse un match, donc on a joué, moi j’ai joué avec eux, donc ils étaient contents. On a tous joué ensemble et ça rappelait des souvenirs de quand on était petit aussi. C'était bien. Donc ouais c'est vrai que bon, quand ils parlent de foot, c'est vrai qu'ils en parlent beaucoup dans leur lycée à des copains et tout ça. Comme quoi ils connaissent quelqu'un qui joue à X (le club étudié) et tout donc... ils sont fiers quoi, ils sont contents » [17 ans, interne]. Son récit montre aussi, à travers l'attente qu'a suscitée sa participation footballistique, le poids de son statut symbolique. D'ailleurs, les parties informelles de football qu'ils entament parfois avec ces « copains » sont des lieux privilégiés d'actualisation de cette place à part, ces jeux constituant une occasion supplémentaire de valorisation. Leur ascension, que marque leur entrée dans un grand club professionnel, transforme donc leur position dans ces réseaux.

De plus, il faut noter que pour les jeunes footballeurs l'entrée dans un mode de vie où domine l'ascétisme sportif, avec la raréfaction du temps disponible qui l'accompagne et souvent l'éloignement spatial, implique une certaine mise à distance des relations antérieures de sociabilité. En matière amicale, les contraintes de la

14/ Leur analyse indique un glissement des différentes influences au profit des liens amicaux (Lahire B., 2004, 500) et une plus grande autonomisation à l'égard des prescripteurs scolaires et familiaux qui se matérialise par le poids des « loisirs-divertissements » (boîtes de nuit, spectacles sportifs, etc.). Sur l'évolution des pratiques culturelles juvéniles dans le sens d'une autonomie grandissante avec l'âge, on peut se reporter au bulletin statistique (Ministère de la Culture et la Communication, 1999). 

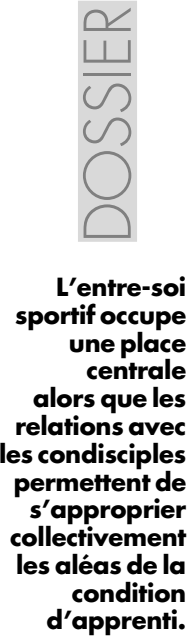

formation, le partage d'une situation commune dans les établissements scolaires et la connivence footballistique tendent à donner aux relations avec les pairs une place centrale (d'autant plus capitale que les enquêtés sont internes). Les bancs de l'école n'ont pas pour eux le même rôle de premier réservoir de relations amicales. Alors que la scolarité joue un rôle central dans la sociabilité « ordinaire » de cette classe d'âge (Pan Ké Shon, 1998), ici, la fréquentation d'un collège puis d'un lycée n'a produit que très peu de relations sociales durables et associées à des activités de loisirs. L'entre-soi sportif occupe une place centrale alors que les relations avec les condisciples permettent de s'approprier collectivement les aléas de la condition d'apprenti. Comme le souligne Bruno Papin au sujet des gymnastes, « en limitant l'aire sociale de fréquentations possibles » les structures de haut niveau «contribuent à limiter les déviances possibles» (Papin, 2000) et facilitent ainsi l'incorporation des contraintes ${ }^{15}$. Si la formation favorise les relations sportives, ses contraintes et leur intériorisation (par l'intermédiaire du souci de la préservation du corps) contribuent également à limiter les activités de loisirs associées à la sociabilité. Ce sont en particulier les privations d'activités les plus associées à leur classe d'âge (comme les sorties nocturnes) ${ }^{16}$ qui leur donne très souvent le sentiment de "passer à côté de leur jeunesse ». L'apprentissage les place dans une situation de contrainte spécifique face aux sollicitations de leur réseau de sociabilité et leur désir de participation à ces activités ne peut, généralement, s'actualiser que de façon très contrainte, durant les vacances ou à distance des échéances sportives. Ils regrettent régulièrement ce qu'ils considèrent souvent comme des «sacrifices». Cependant, leurs tiraillements et leur participation, même a minima, à ces sorties ( 8 sur 10 déclarent sortir en bar ou boîte de nuit pendant les vacances) se comprennent d'autant mieux que maintenir l'ascétisme en permanence est rendu difficile par les sollicitations amicales et que l'affirmation virile dans des groupes souvent très masculins passe aussi par cette sociabilité festive. Les relations amicales sont alors au centre du provisoire desserrement des contraintes sportives et offrent une échappatoire, une occasion de sortie de l'emprise exigeante et concurrentielle du centre de formation. Elle fonctionne alors comme un «troisième lieu » (Renahy, 2005), un espace intermédiaire entre le club et les relations familiales qui autorise la sortie intermittente de l'emprise sportive et permet de vivre le temps des vacances comme des moments de relâche.

15/ La restriction des réseaux amicaux impliquée par ce type d'apprentissage a été régulièrement soulignée dans les travaux sur les champions sportifs, (Brissonneau et al., 2008, 161; Fleuriel, 2004, 61).

16/ On sait l'importance des sorties entre amis pour cette tranche d'âge, ainsi $35 \%$ des 15-19 ans déclarent sortir plsieurs fois par semaine et le plus souvent avec des amis (77\%). De plus, la sortie en discothèque et boîte de nuit est caractéristique de cette classe d'âge (Donnat, 1998, 42-47). 
Les relations amicales et les activités qui leur sont associées sont donc fortement marquées par les contraintes sportives : la position occupée parmi les « copains » aussi bien que la nécessaire centration footballistique en témoignent.

\section{Renommée locale, « jalousie » et injonction à la fidélité}

Au-delà des liens d'affinités, les relations sociales liées à l'interconnaissance locale constituent un autre ensemble de rapports qui sont modifiés par l'entrée dans le club professionnel. Ainsi, l'attente et l'espérance parentales déjà relevées sont d'autant plus fortes que la carrière sportive est souvent synonyme de renommée locale. Les performances sportives permettent la constitution d'un capital symbolique dont les apprentis, mais aussi la famille, sont les récipiendaires. Cette reconnaissance de la valeur de leur parcours est d'abord forte dans le cadre sportif: anciens « coachs» ou partenaires sont souvent les témoins privilégiés de cette ascension. Mais cette reconnaissance dépasse très fréquemment le milieu footballistique pour atteindre de manière diffuse le village de résidence ou le quartier d'appartenance. La diffusion de leur consécration sportive a d'ailleurs souvent été relayée par la presse locale (portraits, articles relatant la signature d'un contrat ou la conquête d'un titre, etc.), voire par le bulletin municipal de leur commune qui se fait l'écho de leurs succès sportifs. À l'intérieur d'une aire de voisinage et d'interconnaissance, les enquêtés comme les parents sont souvent interpellés au sujet de leur parcours à l'image de ce que vit le père de l'un d'entre eux: « Je vais acheter ma viande, on me dit "alors et votre fils ce week-end, il a fait quoi?", hein bon. Alors c'est pas partout, dans tous les magasins, mais beaucoup de gens qui savent (...). Souvent d'ailleurs quand on rencontre quelqu'un, on nous demande de ses nouvelles avant de nous en demander à nous [rires] ».

Les relations de proximité spatiale participent donc elles aussi à la construction d'une place singulière pour les jeunes apprentis. Comme au lycée, cette reconnaissance est parfois conflictuelle, d'où les réticences de certains joueurs à la divulgation de leur parcours. Elles s'expliquent par la même ambivalence des effets de leur capital symbolique, ils craignent la manifestation d'une hostilité analogue. Cette appréhension pousse, par exemple, ce jeune résident d'un quartier d'habitat populaire de l'agglomération étudiée, à ne pas exhiber de signes visibles de sa réussite: "Là j'peux acheter une grosse caisse, j'peux frimer, mais là où j'suis là, en plus dans le quartier où je vis, ils brûlent beaucoup les voitures, c'est des jaloux un peu tu vois donc... moi j'suis simple, j'essaie de pas trop me

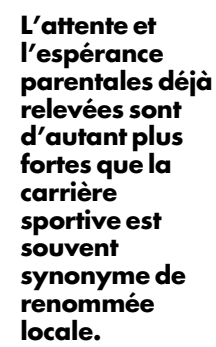


mettre en valeur» [Dimitri, 18 ans, fils d'un ouvrier qualifié]. Ils expriment alors souvent la peur des accusations des « jaloux » leur reprochant d'être «trop fiers » ou d'« avoir la grosse tête». C'est ce dont témoigne également la mère de Dimitri : «Il est resté tel qu’il est mais des fois y a... tu vois des gens de son âge, des jeunes de son âge qui disent "ah mais Dimitri maintenant t'as la grosse tête !" Je lui ai dit: "Ne pense pas à ça, c'est de la jalousie". "Tu n'as même pas changé, tu leur dis bonjour, des fois ils sont en bas tu descends, tu causes avec eux et tu remontes mais pourquoi il te dit ça? Il veut être à ta place, c'est ça. Et il dit ça, c'est pour te décourager ou te choquer" » [mère au foyer, ancienne ouvrière]. Or il faut noter que cette appréhension est distribuée socialement, elle est très souvent le fait d'enquêtés issus des milieux populaires. Deux raisons permettent d'expliquer cette récurrence. D'une part, on peut lier ce constat au fait que, comme nous y invite une série de travaux (Retière, 2003; Renahy, 2010), ce sont dans les familles de ces enquêtés que le capital d'autochtonie, entendu comme le capital social et symbolique de la famille et de ses membres dans un espace de relations localisées, forme une composante importante de la vie sociale. Ainsi, la participation du parcours sportif à la réputation familiale au sein des relations sociales locales a des chances d'être une composante importante des ressources familiales. D'autre part, les apprentis d'origine populaire sont plus souvent confrontés à une injonction à la fidélité (ne pas « trahir »), fréquemment relevée dans l'analyse des transfuges issus des classes populaires (Hoggart, 1991 (1988)) et qui agit comme un rappel à l'ordre ${ }^{17}$. Il est révélateur, a contrario, que pour les enquêtés aux origines sociales plus élevées, la crainte de la «jalousie » soit souvent absente. C'est que l'accès au monde footballistique leur pose, pourrait-on dire, d'autres questions et en particulier celle de trouver la reconnaissance de la valeur de leur parcours au sein de leur famille quand celui-ci entache la scolarité. Pour ce fils de parents médecins par exemple, le problème central semble avoir été celui de sa non-conformité scolaire aux normes familiales:

[Enquêteur] «- Est-ce que tu ressembles à quelqu'un dans ta famille?

- Non. Non. Ni physiquement, ni mentalement, ni dans ce que j'ai fait.

17/ Florence Weber a, en particulier, souligné combien les accusations croisées de « fierté » (« s'y croire », « se la péter», etc.) et de «jalousie » sont fréquentes en milieu ouvrier en raison de l'« exigence d'égalité » (Weber, 1989, 79) qui structure les relations d'interconnaissance et qui prolonge le poids du « conformisme populaire » (Weber, 1989, 181) mis en évidence par Richard Hoggart. C'est ce qui explique, selon elle, « les accusations croisées de fierté (tendance centrifuge qui peut mener à l'exclusion) et de jalousie (tendance centripète par la réaffirmation de la co-appartenance). L'une comme l'autre poussent ceux qui veulent se maintenir "à l'intérieur" à "rester dans le rang" » (Weber, 1989, 179). 
- Ils se demandent comment ça se fait? [rires].

- Ouais ils se demandent. Non, mais dans ma famille tous ont privilégié les études, moi non, ben déjà là je me suis mis en retrait de tout le monde. Ça eu du mal des fois à passer, mais surtout au début. Quand tu vas annoncer à tes grands-parents "ah mais moi les études c'est pas ce qui m'importe". Ils comprennent pas, ils se disent que les études c'est le seul moyen de réussir dans la vie et que si tu décides d'arrêter c'est fini quoi. Ils ont eu un peu du mal à comprendre » [apprenti de 19 ans, a échoué aux épreuves du baccalauréat ES]

En revanche, pour une partie importante des enquêtés issus de milieux populaires, c'est la crainte récurrente d'être l'objet de jalousies, comme leur peur d'être désigné comme «fier» qui dominent. Ainsi, la fidélité à son passé à travers l'attachement maintenu aux amis et relations de voisinage semble être un sujet d'autant plus sensible que les jeunes footballeurs sont issus des milieux populaires et qu'ils doivent souvent répondre à la double injonction de « réussir » sans « trahir ». À l'image des lycéens issus d'un quartier populaire étudiés par Stéphane Beaud (Beaud, 2002), ces apprentis footballeurs vivent la menace de l'accusation de la «trahison » alors que leur investissement footballistique implique une certaine mise à distance de ces attaches locales.

\section{CONCLUSION : UN TRAVAIL DE MISE EN COHÉRENCE ET SES CONDITIONS SOCIALES}

L'engagement des apprentis est intense et exige un haut degré de persévérance. Mobilisés par l'organisation sportive, leur investissement se construit également à l'intérieur des différents réseaux de relations extra-sportives et dans l'interaction entre la carrière sportive et ces univers. Leur parcours sportif agit sur leurs relations familiales, de sociabilité et de voisinage comme il modifie leur place dans l'espace scolaire. Au final, il apparaît que la production de la socialisation sportive peut être définie, en grande partie, comme un travail progressif d'articulation et de mise en cohérence des différents espaces de relations auxquels appartiennent les appentis. Sur ce point, il est possible de comparer la formation sportive à ces familles qui tentent de restreindre la pluralité des espaces de socialisation de leurs enfants (par le contrôle des fréquentations ou le choix d'un établissement scolaire par exemple (Darmon, 2006, 66) pour mieux en assurer la convergence éducative. Ainsi, la prise en charge extensive des joueurs par le club freinent les investissements dans les autres sphères d'activités (scolaire et de sociabilité en particulier) et limite les 
influences contradictoires. Par l'occupation intensive des jeunes, leur prise en charge matérielle et, de manière générale, par l'intériorisation de la priorité des enjeux footballistiques, la formation assure une mise en cohérence des espaces d'appartenance. Se manifeste ainsi la «tendance à l'hégémonie » (Castel, 1968) d'une institution qui n'est jamais autant visible que lorsqu'elle se heurte à la concurrence d'autres univers d'appartenance (les tensions existantes avec les familles et avec le responsable pédagogique sont les témoins de cette concurrence éducative entre le club et les familles d'un côté, l'école et le club d'un autre). Cette mise en convergence possède également une seconde facette : la socialisation sportive se joue au croisement des différents groupes et instances de socialisation en ce que ces espaces contribuent, chacun à leur manière, à produire une "place à part » pour les apprentis. Les actes réitérés de reconnaissance de leur ascension sportive dans des scènes sociales différentes deviennent un élément de la consolidation de l'attachement à l'investissement sportif. Cette place à part a d'autant plus de chances d'être intériorisée qu'elle est construite dans des espaces de relations variés (famille, liens amicaux, relations d'interconnaissance locale) au sein desquels leur capital symbolique distinctif les assigne de manière répétée.

Si une bonne partie de la socialisation sportive peut être décrite comme un travail de mise en cohérence des appartenances des apprentis, il faut rappeler que la force d'emprise du centre de formation et l'articulation entre les différentes scènes n'est pas indépendante de la position sociale des individus. Et, pour le dire autrement, la présentation successive des différentes espaces d'appartenance ne doit pas faire oublier que l'instance familiale joue un rôle premier et central dans la socialisation des jeunes apprentis et, à travers elle, l'appartenance à un milieu social. Il existe des conditions sociales de réussite du travail de mise en cohérence et l'analyse diachronique des différents espaces contribue à rendre raison du recrutement social de cette population. Ainsi, la place majoritaire des enfants des classes populaires peut se comprendre, notamment, par le fait que la formation a plus de chances de trouver un terrain favorable au travail de mise en convergence des investissements. En particulier, l'apprentissage au sein du Centre a moins de chances de heurter les dispositions parentales en matière de scolarité. A contrario, à l'intérieur des familles plus fortement dotées en capitaux économiques et culturels, les contraintes sur la scolarité sont plus souvent un frein à l'engagement, risquant de faire moins facilement de la professionnalisation un horizon souhaitable. De plus, pour les familles des classes populaires l'engagement sportif est à l'origine de ressources économiques et symboliques (en termes de reconnaissance locale en particulier) qui ont de fortes probabilités de jouer un rôle significatif auprès de celles-ci. 
Cette convergence avec les pratiques de la formation sportives a des chances d'être plus facilement réalisée dans ce contexte sachant que, de surcroît, la pratique du football occupe une place traditionnellement forte au sein des cultures populaires masculines.

\author{
Julien Bertrand \\ École Normale Supérieure de Lyon / \\ Centre Max Weber (UMR 5283 CNRS) \\ bertrandju@aliceadsl.fr
}

\title{
RÉFÉRENCES BIBLIOGRAPHIQUES
}

BEAUD S., 2002

BERTRAND J., 2008

BERTRAND J., 2009

BERTRAND J., 2009b

BIDART C., 1997

BOURDIEU P., 1980

BOURDIEU P., 1982

BRISSONNEAU C., AUBEL O., OHL F., 2008

CASTEL R., 1968

DARMON M., 2006

DONNAT O., 1998

FAURE J.-M., SUAUD C., 1999

FAURE S., 2000

FLEURIEL S., 2004

HOGGART R., 1991 [1988]
$80 \%$ au bac... et après ? Les enfants de la démocratisation scolaire. Paris : La Découverte.

La Fabrique des footballeurs. Analyse sociologique de la construction de la vocation, des dispositions et des savoir-faire dans une formation au sport professionnel. Doctorat de Sociologie, Université Lyon 2.

Entre « passion » et incertitude : la socialisation au métier de footballeur professionnel. Sociologie du travail, vol 51, $\mathrm{n}^{\circ}$ 3, p. 361-378.

Sociabilité et vocation sportive. Des apprentis footballeurs parmi leurs pairs. In : Guibert C., Loirand G. and Slimani $\mathrm{H}$.

Le Sport entre public et privé : frontières et porosités. Paris : L'Harmattan, p. 109-117.

L’Amitié, un lien social. Paris : La Découverte.

Le Sens pratique. Paris : Minuit.

Les rites comme actes d'institutions. Actes de la recherche en sciences sociales, $\mathrm{n}^{\circ} 43$, p. 58-63.

L'Épreuve du dopage : sociologie du cyclisme professionnel. Paris : PUF.

Présentation. In Goffman E., Asiles : études sur la condition sociale des malades mentaux. Paris : Minuit.

La Socialisation. Paris : Armand Colin.

Les Pratiques culturelles des français. Enquête 1997. Paris : La Documentation Française.

Le Football professionnel à la française. Paris : PUF.

Apprendre par corps. Socio-anthropologie des techniques de danse. Paris : La Dispute.

Le Sport de haut niveau : sociologie d'une catégorie de pensée. Grenoble : PUG.

33 Newport Street. Autobiographie d'un intellectuel issu des classes populaires anglaises. Paris: Hautes Études-Gallimard-Le Seuil. 
LAHIRE B., 2004

LEFÈVRE N., 2007

MINISTÈRE DE LA CULTURE ET LA COMMUNICATION, 1999

PAN KE SHON J., 1998

PAPIN B., 2007

REGNIER F., LHUISSIER A., GOJARD S., 2006

RENAHY N., 2005

RENAHY N., 2010

RETIÈRE J.-N. 2003

SCHWARTZ O., 1995

SLIMANI H., 2000

SORIGNET P., 2004

SUAUD C., 1978

WACQUANT L., 1989

WAGNER I., 2004

WEBER F., 1989
La Culture des individus. Paris : La découverte.

Le Cyclisme d'élite français : un modèle singulier de formation et d'emploi, thèse pour le Doctorat de Sociologie, Université de Nantes.

Les loisirs des 8-19 ans. Développement culturel, n 131 , p. 1-8.

« D'où sont mes amis venus ?... ». INSEE Première, nº 613.

Conversion et reconversion des élites sportives. Paris :

L'Harmattan.

Sociologie de l'alimentation. Paris : La Découverte.

Les Gars du coin. Enquête sur une jeunesse rurale. Paris : La Découverte.

Classes populaires et capital d'autochtonie. Genèse et usages d'une notion, Regards sociologiques, $n^{\circ} 40$, p. 9-26.

Autour de l'autochtonie. Réflexions sur la notion de capital social populaire. Politix, vol. 16, n 63, p. 121-143.

L'empirisme irréductible. In Anderson N., Le Hobo sociologie du sans-abris. Paris : Nathan. p. 265-308.

La Professionnalisation du football français : un modèle de dénégation. Doctorat de sociologie, Université de Nantes.

Sortir d'un métier de vocation : le cas des danseurs contemporains. Sociétés contemporaines, $\mathrm{n}^{\circ}$ 56, p. 111-132.

La Vocation. Conversion et reconversion des prêtres ruraux. Paris : Minuit.

Corps et âmes : notes ethnographiques d'un apprenti boxeur. Actes de la recherche en sciences sociales, $\mathrm{n}^{\circ} 39$, p. 67-55.

La formation des violonistes virtuoses : les réseaux de soutien. Sociétés contemporaines, n 56, p. 133-163.

Le Travail à-côté : étude d'ethnographie ouvrière. Paris : INRA-EHESS 